\title{
MANAGEMENT OF FACTORY AUTOMATION CONTROLLERS WITH PEER-TO-PEER TECHNOLOGY
}

\author{
Satoshi IWATSU and Akio NODA \\ Advanced Technology R\&D Center, Mitsubishi Electric Corporation \\ 8-1-1, Tsukaguchi-Honmachi, Amagasaki, Hyogo 661-8661, JAPAN \\ e-mail: \{Iwatsu.Satoshi, Noda.Akio\}@wrc.melco.co.jp
}

\begin{abstract}
P2P (Peer-to-Peer) technology spread explosively as a method of exchanging the music data etc. on the Internet for a certain period of time. We think about its application to the FA (Factory Automation) field. When the configuration of FA controllers connected on the network is changed, the parameters of them or of their applications might have to be recreated. UPnP (Universal Plug and Play) is one of the implementation methods of the automatic setting that P2P requires. Trouble of complex settings could be saved by installing the function of UPnP in FA controllers.
\end{abstract}

\section{INTRODUCTION}

In the Internet world, P2P technology is recently in the limelight. Peers can automatically connect and interact with each other in P2P, having functions such as to discover other peers and to advertise their own existence or capabilities. Originally, P2P related software was developed so that many anonymous individuals can exchange data directly. One of the famous or notorious applications is exchange of music data. P2P takes it a presupposition that the number of peers changes dynamically. Therefore, it can be available for automatic configuration of network systems in the intranet. UPnP, which Microsoft advocates, is one of those technologies which aim that.

On the other hand, users of FA applications are suffering from complex parameter settings needed to connect them with FA controllers. Since the production style has been shifted to a diverse-types-and-small-quantity production, network configurations of FA controllers came to change more frequently.

We think it effective to apply UPnP-based communication methods to FA controllers and FA applications. Users can run the system without knowledge of the network. We have implemented some of those methods on personal computers each of which plays the role of a dummy FA controller or an FA application. In this paper, we show effectiveness thorough the demo program.

\section{P2P AND UPnP}

\subsection{P2P}

A communication method where a terminal in the network might play either role of the server or the client is called $\mathrm{P} 2 \mathrm{P}$. The terminal is called peer and is in the equal relation to others in the network. A method of the file exchange in the Internet such as Napster and Gnutella is often enumerated as an example of P2P. They differ by structural models. A central server manages the list of files available in the Napster type. Data are transmitted one by one in the Gnutella type. They can be represented as Hybrid P2P and Pure P2P respectively.

It is thought that the function to set the system of the network or the application automatically is indispensable for P2P. This function is effective when data are interactively exchanged between terminals in the inside and outside of the LAN and when the terminal that uses a certain application is changed or added. UPnP is one of the mechanisms that the automatic setting is achieved.

\subsection{UPnP}

$\mathrm{UPnP}$ is a specification of the plug-and-play technology on the network appliance [1]. In the framework of UPnP, appliances on the network automatically recognize each other, advertise services others can utilize, and search for services others are providing. They come to be able to use services of each other by these functions.

In UPnP, appliances are classified into 2 types by their roles [2]. One is "device", which is the appliance that provides others its services. The other is "control point", which is the appliance that uses services of devices.

There are 6 basic operations in UPnP as follows.

\section{i. Addressing}

When an appliance is connected with LAN, it broadcasts a request to set its IP address etc. and adopts the settings assigned by a DHCP (Dynamic Host Configuration Protocol) server if exists.

ii. Discovery

Appliances notify their own existence by the multicast on UDP (User Datagram Protocol). Devices notify their services (advertisement), and control points retrieve services (search).

iii. Description

Once control points recognize a device, they request it to send detail information by unicast. Contents are described with XML and sent by HTTP protocol.

iv. Control

Control points operate devices by using SOAP (Simple Object Access Protocol). 


\section{v. Eventing}

Control points which monitor events of a device need to be registered in its subscription list. When a device detects a trouble etc. it notifies control points in the list.

vi. Presentation

Control points can retrieve the device presentation page at the URL it notifies, and load it into a browser. Users can control it or view its status.

\section{SYSTEM MODEL}

When users want to communicate with an FA controller to manage, parameters of the management application might have to be set based on the configuration of the network. In the case of Mitsubishi's FA controllers, for example, users set the type name of the controller, the IP. address, etc. as the parameters of EZSocket, which is the middleware for them. This is one of the reasons why it is difficult to set up network based application systems or to deal with dynamic changes of system configuration. Referring to the specification of UPnP, we designed an automatic configuration function for management of FA controllers on the network to avoid that. We assume that our demo system consists of several FA controllers, a personal computer with a management tool and a DHCP server all of which are connected each other by Ethernet. Fig. 1 shows the structure of our demo system. On personal computers, we implemented function modules of FA controllers and a management tool of them.

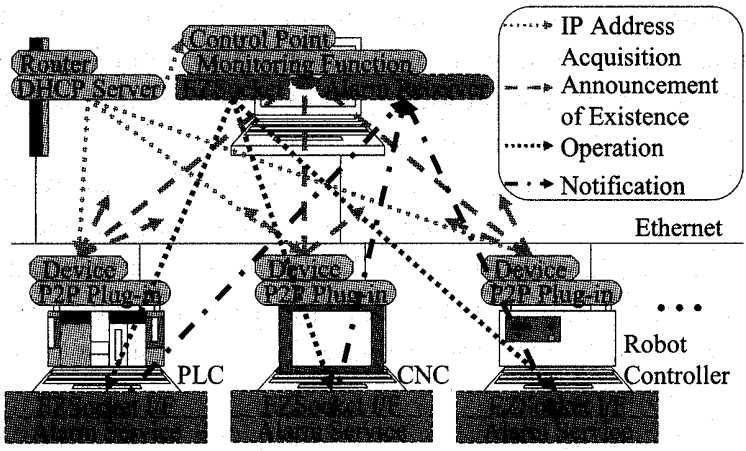

Fig. 1 System structure on the network

Each of FA controllers on the network advertises its own existence by multicasting using UDP and a management tool discovers their existence by listening to the multicast. An FA controller advertises its controller type, a URL to services it provides and so on. That URL includes an IP address automatically assigned by a DHCP server. On the side of a management tool, connected FA controllers are listed up. Since they advertise their own existence periodically, it can notice whether they are active or not. Each line of the list includes the details of what each FA controller advertises. This information enables a management tool to make interaction with them. Concretely, they store EZSocket's connection parameters described in an XML file where the URL points, which enable it to make operation such as monitoring by EZSocket's API (Application Program Interface).

\section{DEMO IMPLEMENTATION}

\subsection{Plug-in for Device}

On the device side, an advertisement function the device notifies its own information is required. When the program starts up, a thread for advertisement is created and it begins multicasting message of its information periodically. It includes the expiration time so that control points can judge whether it is alive or not.

As control points request its detail data by HTTP, it also creates a thread for a simple HTTP server function. We adopt EZSocket's parameters for the detail data.

\subsection{Monitoring Application for Control Point}

On the control point side, a function for detecting the devices sending their information is required. The program has simple GUI to list up FA controllers as devices. Each column of the list corresponds to the type of the FA controller, the IP address, the communication port and the path for detail data. Since devices notify those data, users do not have to set them manually.

When an FA controller is detected by its advertisement, the data mentioned above are indicated at the new row of the list. If it does not send data by its expiration time, the control point judges it disconnected and marks on its row of the list. If it reconnects, the mark disappears.

\section{CONCLUSION}

It saves trouble in setting up management software and makes progress in scalability or robustness for dynamic change of configuration of FA controllers to implement P2P-applied plug-ins to FA controllers. Indeed, we feel it easy to set up the demo system, because we have only to connect appliances each other by Ethernet and to start them up. We also made sure that other $\mathrm{P} 2 \mathrm{P}$ related application which uses a different multicast channel can run with this demo system simultaneously. We think program codes implemented on each appliance are small enough to transplant to embedded systems. Farther, we consider implementing treatment of events regulated in UPnP. Though a management tool as a client has usually needed to poll an FA controller as a server, in this case their role are reversed and an FA controller informs a management tool of the event when it occurs. Both an FA controller and a management tool can play either role of a server or a client. This is one of the characteristics of P2P. As described above, we found it effective to apply P2P technology to FA systems.

\section{REFERENCES}

[1] http://www.upnp.org/

[2] Nuriya, T., UPnP Has Come (in Japanese), Nikkei Network 2002.06, Nikkei BP, 2002, pp. 70-77. 